\title{
Coalitions of Things: Supporting ISR Tasks via Internet of Things Approaches
}

\author{
Alun Preece ${ }^{a}$, Ian Taylor ${ }^{a}$, Andrew Dawson $^{a}$, \\ Dave Braines ${ }^{b}$, Nick O'Leary ${ }^{b}$, Anna Thomas ${ }^{b}$, Richard Tomsett ${ }^{b}$, \\ Tom La Porta ${ }^{c}$, \\ Jonathan Z. Bakdash ${ }^{d}$, Erin Zaroukian ${ }^{d}$ \\ ${ }^{a}$ Crime and Security Research Institute, Cardiff University, Cardiff, UK \\ ${ }^{b}$ Emerging Technology Services, IBM United Kingdom Ltd, Hursley Park, Winchester, UK \\ ${ }^{c}$ School of Electrical Engineering and Computer Science, Penn State University, USA \\ ${ }^{d}$ Human Research and Engineering Directorate, US Army Research Laboratory, Aberdeen \\ Proving Ground, USA
}

\begin{abstract}
In the wake of rapid maturing of Internet of Things (IoT) approaches and technologies in the commercial sector, the IoT is increasingly seen as a key 'disruptive' technology in military environments. Future operational environments are expected to be characterized by a lower proportion of human participants and a higher proportion of autonomous and semi-autonomous devices. This view is reflected in both US 'third offset' and UK 'information age' thinking and is likely to have a profound effect on how multinational coalition operations are conducted in the future. Much of the initial consideration of IoT adoption in the military domain has rightly focused on security concerns, reflecting similar cautions in the early era of electronic commerce. As IoT approaches mature, this initial technical focus is likely to shift to considerations of interactivity and policy. In this paper, rather than considering the broader range of IoT applications in the military context, we focus on roles for IoT concepts and devices in future intelligence, surveillance and reconnaissance (ISR) tasks, drawing on experience in sensor-mission resourcing and human-computer collaboration (HCC) for ISR. We highlight the importance of low training overheads in the adoption of IoT approaches, and the need to balance proactivity and interactivity (push vs pull modes). As with sensing systems over the last decade, we emphasize that, to be valuable in ISR tasks, IoT devices will need a degree of mission-awareness in addition to an ability to self-manage their limited resources (power, memory, bandwidth, computation, etc). In coalition operations, the management and potential sharing of IoT devices and systems among partners (e.g., in cross-coalition tactical-edge ISR teams) becomes a key issue due heterogeneous factors such as language, policy, procedure and doctrine. Finally, we briefly outline a platform that we have developed in order to experiment with human-IoT teaming on ISR tasks, in both physical and virtual settings.
\end{abstract}

Keywords: coalition operations; intelligence, surveillance and reconnaissance; internet of things

\section{INTRODUCTION}

The Internet of things (IoT) is the inter-networking of physical objects embedded with computational capability and network connectivity, enabling the objects to collect, process and disseminate data. ${ }^{1,2}$ IoT technologies are expected to be transformative in a wide variety of domains including smart cities, health care, and smart homes. Currently, there are significant concerns around security issues in the IoT context, reminiscent of the early period of the Web and electronic commerce. However, it is reasonable to expect improved security approaches - as with HTTPS and SSL in electronic commerce - will alleviate these problems, opening up a rapidly-growing space of new applications.

Recently, attention has turned to prospects for IoT technology take-up in military domains. ${ }^{3}$ Many potential applications can be extrapolated from analogous commercial and social domains, including personal sensing to

Send correspondence to A.Preece, E-mail: PreeceAD@cardiff.ac.uk, Tel: +44 (0) 2920874653 
monitor the health and wellbeing of military personnel, logistics management, and crowdsensing approaches. However, a key domain for military IoT application is in supporting intelligence, surveillance and reconnaissance (ISR) tasks. Internet-enabled objects intrinsically perform sensing, processing, and dissemination operations, so are a natural paradigm for ISR support.

Moreover, in at least the U.S. and UK military contexts, IoT has been highlighted as a key element of future operations. One driver for this is the desire to reduce the deployment of human personnel in front-line situations, not least for cost reasons. ${ }^{4}$ Other drivers place IoT technologies in a central position in U.S. and UK military strategy. For example, technologies including robotics and autonomous systems were identified in 2014 as key enablers of the U.S. Department of Defence 'Third Offset' initiative* while, in the UK, IoT technologies are highlighted as significant elements of 'Warfare in the Information Age' concept. ${ }^{5 \dagger}$

Modern military operations often feature coalitions in which multiple partners cooperate to achieve mission objectives. ${ }^{6}$ The coalition operations environment is characterized by a high degree of dynamicity, a need for effective information and ISR asset sharing among partners, especially at the tactical edge, and constraints dictated by organizational and mission policies. ${ }^{7}$ The potential for deployment of IoT assets into this space is high, but there are significant open questions on how to harness this potential in a highly heterogeneous environment.

This paper is organized as follows: Section 2 considers the potential applications of IoT technology for ISR tasks in terms of the affordances of things; Section 3 considers issues in harnessing IoT technology for ISR in the coalition context; Section 4 takes account of human-computer collaboration (HCC) factors in IoT adoption for ISR in coalition missions; Section 5 introduces a prototype platform for conducting human-in-the-loop experiments with simulated ISR assets; Section 6 concludes the paper.

\section{AFFORDANCES OF THINGS}

The notion of affordances has been central to the human-computer interaction (HCI) field for many years, referring to what an object 'is for', i.e., 'the perceived and actual properties of the thing, primarily those fundamental properties that determine just how the thing could possibly be used' ${ }^{8}$ In the ISR context, it is necessary to classify IoT devices in terms of their affordances to a range of ISR tasks. For example, previous work has examined affordances in the scope of visual analytics, focusing on the following: ${ }^{9}$

- large-scale data manipulation;

- collection and storage of large data volumes;

- efficient data movement;

- bias-free analysis.

In this context it is usual to also consider the classic HABA-MABA (humans are better at vs machines are better at) task characterization. While dated, this paradigm of human supervisory is still the dominant approach to human-machine interaction, and the purpose of HCC systems is to aim for each party to exploit their strengths and compensate for the weaknesses of the other. ${ }^{10}$ Complementary to the above examples, which represent instances of MABA, the following HABA cases can be identified (based on current machine capabilities): ${ }^{9}$

- visual and audiolinguistic perception;

- sociocultural awareness;

- creativity;

- broad background domain knowledge.

\footnotetext{
*https://web.archive.org/web/*/https://www.defense.gov/News/Speeches/Speech-View/Article/606635

${ }^{\dagger}$ Potential use of IoT by adversaries is also an important topic, but beyond the scope of this paper.
} 
To be useful in an ISR context, IoT technologies need to offer affordances that sit comfortably within the MABA domain, complementing and enhancing the abilities of human operatives (i.e., the focus is on HCC, not human replacement by automation). A provocative alternative perspective views IoT devices kinds of 'enchanted' object, where the IoT-enablement of familiar objects affords their user additional 'powers' ${ }^{11}$ An ISR-relevant example is Bilbo and Frodo Baggins' sword, 'Sting', in J.R.R. Tolkein's The Hobbit and The Lord of the Rings, which is not only a weapon but is also, thanks to its ability to glow when enemies are nearby, a situation awareness device. One significant point here for IoT design is that things may reveal their affordances in ways very different to those typically expected from computers or robot systems, and this opens up a wide range of possibilities for innovation in future ISR systems. A second significant point is that IoT approaches offer new opportunities to overcome classic HCI issues and strive for what has been termed a 'zero training overhead' (see also Section 4).

Given that ISR is intrinsically linked to sensemaking, ${ }^{12}$ the ISR affordances of things will also in many bases relate to areas of human augmented cognition. ${ }^{13}$ The capabilities of IoT devices may be conceptualized as enhancing human operatives' cognitive abilities such as:

- memory, e.g., a database-connected heads-up display able to recall, Google Now-style ${ }^{\S}$, context-aware information from a mission knowledge base of both long term and short term intelligence;

- perception, e.g., a body-worn camera equipped with image processing sufficient to detect anomalous objects that the wearer's cognitive biases may tend to cause them to miss;

- reasoning, e.g., a tactical drone equipped with models of current and past missions, sufficient to perform ISR proactively in anticipation of likely threats;

- communication, e.g., a smart watch able to alert both its wearer - via context-sensitive modalities such as speech, haptics, or visual display - and also the wearer's team members of essential elements of mission information.

These new modalities of interaction between IoT devices and humans increase the opportunities for ambient awareness where information is imparted in ways which can increase awareness without requiring explicit attention. From this perspective, viewed as cognitive aids, IoT devices need to be not merely context-aware as in the case of commercial offerings like Google Now, but mission-aware in that they hold some knowledge of what (ISR) tasks they are aiming to help the mission accomplish.

Returning to the above discussion of MABA-type machine affordances for ISR, we can summarize IoT-specific capabilities as follows:

Large-scale data manipulation The key IoT characteristic here is how the increasing numbers of networked objects in the field not only generate data with unprecedented volumes, velocity and variety, ${ }^{14}$ they also provide new opportunities for processing and exploiting the data closer to the network edge. ${ }^{15}$

Collection and storage of large data volumes Increasing storage as well as data collection capabilities of networked objects opens up an array of new distributed system architectures, particularly where (a) local processing and storage is less constrained than network bandwidth, favouring local processing and communication of (less) processed rather than (more) raw data; and (b) constraints on data sharing between coalition partners entail preferences for decentralized information architectures.

Efficient data movement Following from the above discussion, the emphasis in ISR system design in an IoT context will shift from a primary focus on processing (what products to derive from data) to a primary focus on communication (what needs to be shared between things at various levels of locality and globality). ${ }^{16}$

\footnotetext{
${ }^{\ddagger}$ http://web.archive.org/web/*/http://techcrunch.com/2012/10/05/building-for-the-enterprise-the-zero-overheadprinciple-2/

${ }^{\S}$ https://web.archive.org/save/https://en.wikipedia.org/wiki/Google_Now
} 
Bias-free analysis This is arguably the most interesting and challenging area in which IoT approaches can have an impact on ISR practice. Considerable work has been done in the area of supporting analysis and mitigating human biases in the intelligence analysis process ${ }^{17}$ but most studies focus on analysis at higher levels and over longer time frames than tactical intelligence. So more forecasting of future events months or even years away.the focus tends to be on analysts near the centre of the network rather than supporting tactical intelligence in field settings. As noted above, a growing prevalence of IoT devices in the field may enhance operatives' cognitive abilities in terms of memory, perception, attention, and communication.

Having considered affordances of IoT devices for ISR tasks, the following section focuses on IoT deployments for ISR in a coalition operations context.

\section{COALITIONS OF THINGS}

Previous work in the area of managing ISR assets for coalition operations has extensively considered the problem of assigning sensors to mission tasks in a highly dynamic context. ${ }^{18}$ Drawing on research in human-robot teams and in particular multi-robot task allocation (MRTA), ${ }^{19}$ multi-sensor task allocation (MSTA) considers four problem dimensions: ${ }^{20}$

Single-task (ST) vs multi-task (MT) sensors: differs in whether a sensor is exclusively assigned to a task or can be shared between multiple tasks.

Single-sensor (SS) vs multi-sensor (MS) tasks: differs in whether the needs of a task can be fulfilled with a single sensor or whether multiple sensors are required.

Instantaneous assignment (IA) vs time-extended assignment (TA): differs in whether the problem requires consideration of the time dimension, i.e., scheduling of sensor-task assignment.

Homogeneous sensor network (HO) vs heterogeneous sensor network (HE): differs in whether the sensor network is composed entirely of a single type of sensor or devices of multiple types.

IoT devices fit into this framework being essentially sensor-equipped objects. The difference is that the granularity of IoT devices will commonly be finer than robot systems (from where MSTA is derived) and 'traditional' sensor platforms such as aerial and ground vehicles or fixed deployments. Thus, combined IoT devices promise to offer a wider range of capabilities and afford a broader range of potential uses. Current sensors and sensors systems have more limited fixed functionality. Therefore it is reasonable to expect that instances of a 'multi-thing task allocation' (MTTA) problem are more likely to be MS than SS ('single thing tasks' will not be common). Moreover, an IoT-based ISR system is more likely to be HE than HO as the paradigm increases the variety of networked objects in the field.

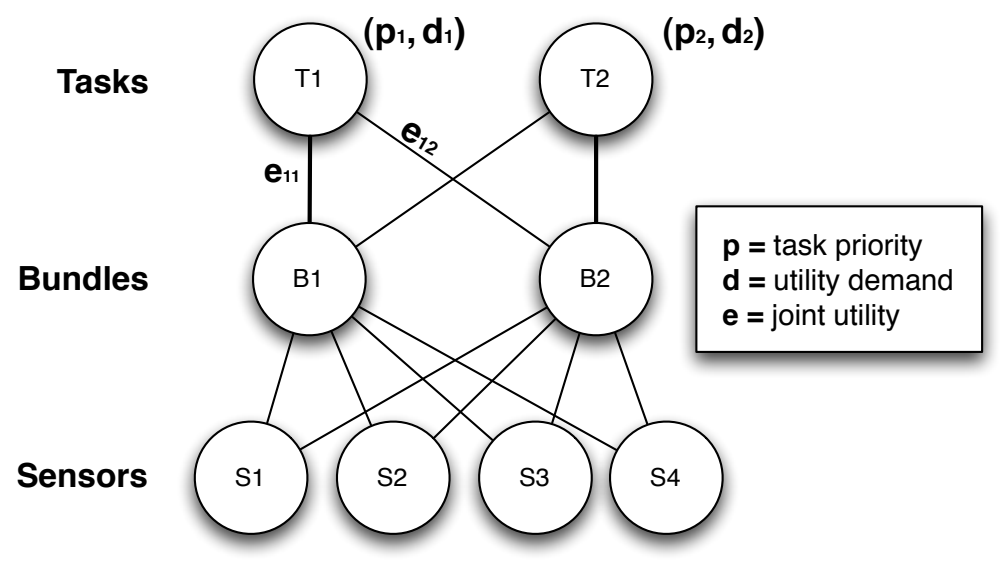

Figure 1. MSTA problem instance ST-MS-IA-HE 


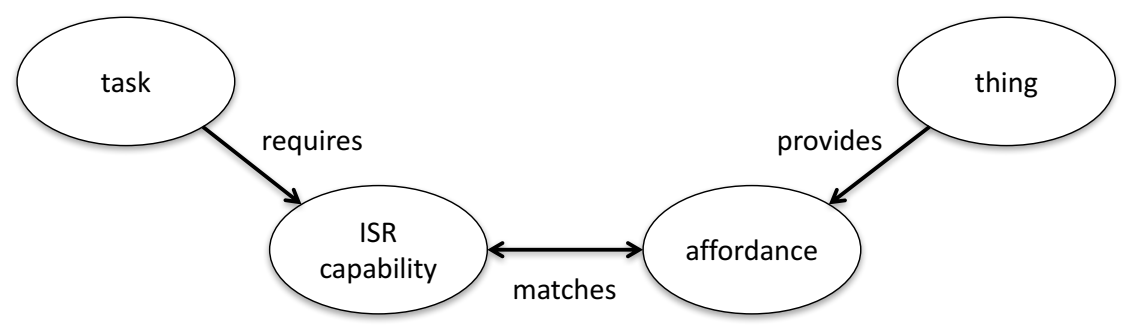

Figure 2. Matching tasks to IoT devices via affordances

MSTA (and hence MTTA) problems can be modelled as tripartite graphs and solved as integer programming problems; ${ }^{20}$ Figure 1 shows the MSTA problem instance ST-MS-IA-HE. The salient features of this model are:

- each task, $T_{i}$, has both a priority, $p_{i}$ (to a mission), and a utility demand, $d_{i}$;

- sensors, $S_{k}$, are grouped into bundles, $B_{j}$, where each bundle has a joint utility, $e_{i j}$, derived from the fitness of that group of sensors to a specific task.

In a coalition context, where assets are shared between partners, there will usually be sharing policies that restrict how sensors can be assigned to bundles and tasks. ${ }^{6}$ Such policies need to be factored-in when defining MSTA instance graphs. ${ }^{18}$ An important consideration is the extent to which knowledge of task assignment and mission policies are pushed to the devices themselves. Giving a device an enhanced degree of mission-awareness, e.g., of the relative priorities of tasks in an MT problem setting, allows a device to make intelligent decisions to self-manage its limited resources such as power, memory, bandwidth, and computation.

Indeed, the key challenge here is not the solving of the graph problem but generating the graph instances, which requires modelling ISR tasks and their requirements, sensors and their capabilities, and asset sharing policies. Previous work approached this as a semantic knowledge representation and reasoning problem ${ }^{18}$ though did not specifically consider sensor capabilities as kinds of affordance as in the previous section. Figure 2 shows this abstract model. While previous work has instantiated this model in terms of 'traditional' sensing systems, how to ground the model for IoT devices in an ISR context will require future work. Moreover, a significant limitation of this model is that it assumes that the demands of ISR tasks are met entirely by sensing devices whereas, as highlighted in the previous section, it is important to consider ISR as a HCC activity. This is the focus of the next section.

\section{HUMAN-THING COLLABORATION}

The 'zero overhead' principle introduced in Section 2 derives from users' growing experience with commercial off-the-shelf (COTS) mobile computing products where software needs to effectively 'introduce itself' to users rather than requiring instruction manuals or training. This has led to raised expectations among users, often unmet by traditional enterprise software. It is reasonable to recognize the same issue in military and ISR systems where users commonly do not possess high degrees of technical skill, training is costly and, especially in the field, personnel are primarily focused on tasks other than operating software.

Widespread familiarity with commercial products such as Apple's Siri ${ }^{\top}$, Amazon's Alexall, Google Now**, and IBM's Watson ${ }^{\dagger \dagger}$ serve to raise user expectation even further in terms of affordances of enterprise systems. This has led to envisioning of data analytics and ISR data-to-decision chains in terms of 'conversations' among humans, devices, and services with communication abilities - Figure $3 .{ }^{16}$ Going even further, recent work has considered the smart home environment in similar terms, but where representations based on natural language (NL) are uniformly used for both human-machine and machine-machine communications. ${ }^{21}$ This provides an environment

\footnotetext{
『https://web.archive.org/web/20160628202009/http://www.apple.com/ios/siri/

"http://web.archive.org/web/20170208020449/https://developer.amazon.com/alexa

${ }^{* *}$ https://web.archive.org/save/https://en.wikipedia.org/wiki/Google_Now

${ }^{\dagger \dagger}$ https://web.archive.org/web/20160628201902/http://www.ibm.com/watson/
} 
where humans and machines can share mission-awareness, e.g., via exchange of tasking requests. Moreover, a conversational approach also naturally allows for push and pull modalities in human-machine communication, e.g., a device will be capable of responding to queries as well as proactively initiating interactions by asking or telling a human or another device. Returning to the notion of 'enchanted' objects from Section 2, it is notable that many such objects in fiction tend to be equipped with NL processing capabilities, e.g., the Queen's mirror in Snow White, and the castle servants in Beauty and the Beast.

A key difference between home and field environments, however, is a relative lack of reliable network (and hence cloud) connectivity in the latter, so current commercial models for intelligent assistants where NL requests are processed in the cloud cannot be directly applied to field settings. Recent work has addressed this problem in terms of lightweight NL processing on mobile devices in 'offline' settings. ${ }^{22}$

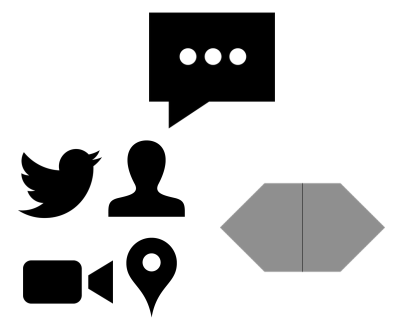

Data sources

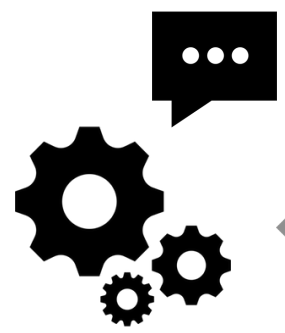

Analytic services

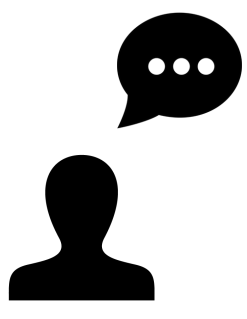

Decision maker

Figure 3. 'Conversational' things in an ISR context

In the ISR context, previous work has examined a number of use cases where such conversational interaction can support HCC, e.g., handling spot reports from patrols, information fusion, and tasking ISR assets. ${ }^{16}$ This work has also experimented with a range of devices including smart phones, ${ }^{20}$ Google augmented reality heads-up displays ${ }^{23}$ (involving the generation of pictorial output as well as language), and smart watches. ${ }^{24}$ While spoken NL input and output is powerful and reduces training overhead, the range of modalities offered by IoT devices presents wider design opportunities. For example, the haptic output common in smart watches allows the device to alert its user silently when this is more appropriate than generating an audible signal.

A further key aspect in communication and collaboration among humans and machines in a coalition context is consideration of information and asset sharing policies. ${ }^{6}$ An advantage of a NL-based approach here is that policies can be expressed in a human-understandable and machine-computable form ${ }^{25}$ and can be pushed to the network edge so that the IoT devices have the necessary policy knowledge as part of their mission-awareness.

Drawing on the asset-task matching approach in Section 3 and the NL-based HCC approach described above, the next section introduces a platform for HCC experiments in the ISR context.

\section{EXPERIMENTAL PLATFORM}

Building on previous research using a crowdsourcing game to simulate tactical intelligence tasks, ${ }^{22}$ this section proposes a simple platform to support online experiments in simulated HCC settings. The platform — Simple Human Experiments Regarding Locally Observed Collective Knowledge (SHERLOCK) — provides an elementary game world consisting of sets of: persons of interest (POIs), locations, objects, sensors (human and machine), and attributes of all of these entities. In a specific instance of a SHERLOCK experiment, participants are provided with a subset of information about the world, for example, a map of locations, 'mugshots' of POIs, and a list of attributes of interest. Their task is to collect specified information, e.g., to discover the location of POIs and the values for their particular attributes, or just to gather as much information as possible. The world is modelled in an NL representation, making it relatively straightforward to define sets of instances for a particular experiment setup.

Worlds have information that is synthetic, natural, or some combination of both. For example, human actors may portray fictional POIs inhabiting real-world locations, or cartoon characters may inhabit synthetic virtual locations. Participants may be tasked to report on natural real-world objects or distinctive objects placed in 


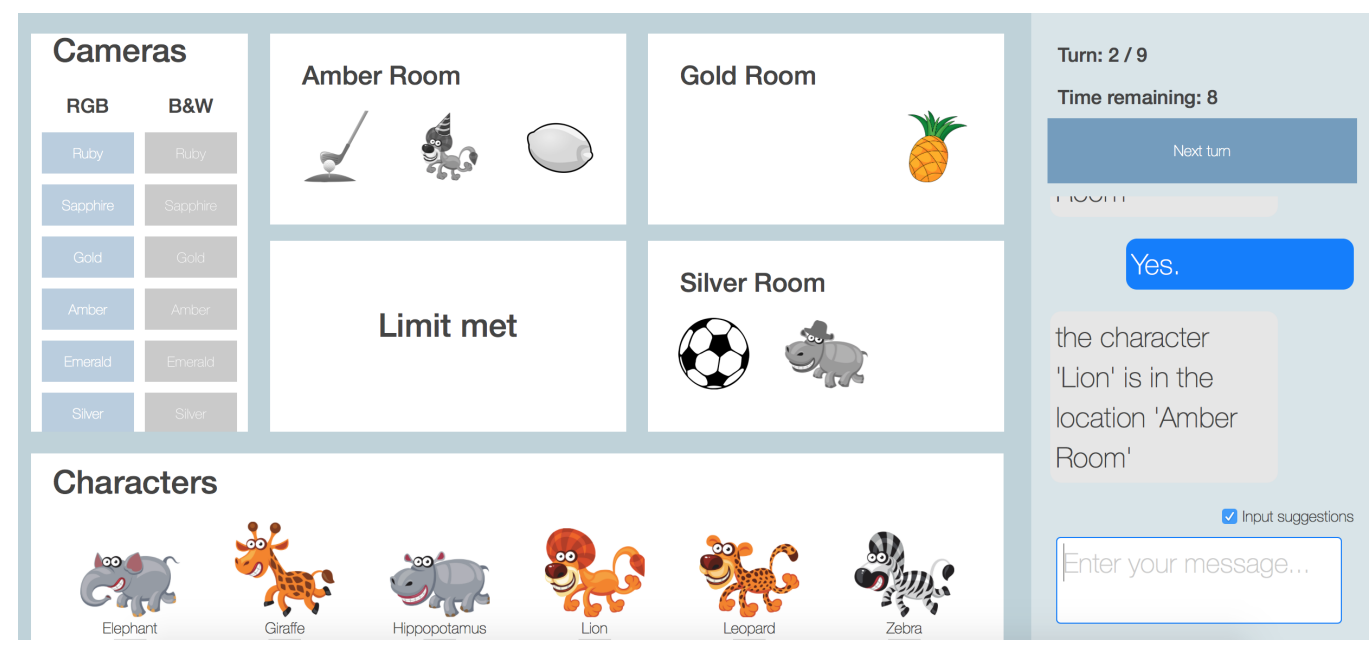

Figure 4. The SHERLOCK+SAM game

locations for the purposes of creating artificial scenes. To avoid challenges with processing NL, synthetic elements are often easier for participants to describe to the agent (with lower complexity and potential for ambiguity). For example, it is easier to describe the location of an object in cases where that location has a label, such as a room number, than it is to describe an 'unlabelled' location, such as a corridor or public space. Highly distinctive objects, such as pieces of fruit or sporting equipment, are also relatively easy to describe.

The SherLock platform includes as a core capability a conversational machine agent that allows human users to impart information (tell a machine agent something), pose queries (ask a machine something), and task assets (delegate some ISR function to a human or machine agent).

Figure 4 shows a screenshot from an online SHERLock setting called SHERLock+SAm ('Sherlock + Sensor Assignment to Missions') designed to support HCC experiments in an ISR context. The game world here involves a number of locations, six of which - the Amber, Gold, Emerald, Ruby, Sapphire and Silver Rooms - can be viewed via simulated cameras. Each room can be viewed via feeds from a colour (RGB) or black and white $(\mathrm{B} \& W)$ camera. There are six POIs, each represented by a cartoon animal: a key to identifying them is at the bottom of the screen. Each POI eats one unique type of fruit, plays one unique sport, and occasionally wears a hat of a unique colour. The centre of the screen provides the user - playing the role of a tactical intelligence operator - with four panels in which they can access a camera feed. Bandwidth limitations mean that there is a limit on how many RGB and B\&W camera feeds that can be accessed simultaneously in a game turn. On the right of the screenshot is the 'chat' interface to the conversational agent, via which the user can report what they see in the feeds during each game turn.

This environment allows a number of variant settings and experimental manipulations:

- The feeds on the left can be chosen automatically by machine using an algorithm similar to the one described in Section 3 (where the machine is aware of what pieces of information are currently unknown and attempts to select feeds that are likely to allow the user to discover that missing information) or manually, or some combination of manual and automatic selection ${ }^{\ddagger}$. Experiments that vary these degrees of control in conjunction with an analysis of the apparent impact on task performance can be used to ascertain the HABA vs MABA position for the camera-selection aspect of this experiment.

- The 'bandwidth' can be varied, allowing different combinations of RGB and B\&W feeds, affecting the relative difficulty of the task; e.g., a B\&W feed is adequate for determining the fruit or sport associated with a POI but not the colour of their hat, as shown in the Amber Room example in the figure (it's striped pink).

\footnotetext{
${ }^{\ddagger}$ i.e., allowing experimentation with alternative levels-of-automation where the ISR system and devices have greater or lesser autonomy. ${ }^{10}$
} 
- The number of turns and turn duration can be varied (see the indicators at the top right).

- The game can be played in single-player or multi-player mode (the latter usually with a shared knowledge base and a means to allow human players to communicate with each other as well as with the machine agents $\left.^{22}\right)$.

Thus far, the core SHERLOCK platform has been extensively tested with over 200 players in crowdsourcing settings $^{22}$ (human sensors only) while the simulated IoT SHERLOCK+SAM setting shown in Figure 4 has been playtested with small groups. Future work will see this environment used for more extensive trials with a range of experimental manipulations, a wider array of simulated IoT devices besides cameras, and more complex sets of network and policy constraints besides simple 'bandwidth' limitations.

\section{CONCLUSION AND FUTURE WORK}

This paper has attempted to look beyond current considerations of the prospects for widespread adoption of IoT approaches in operational environments that tend to focus predominantly on security considerations. The intention has not been to downplay the importance of such considerations but to seek to understand some of the opportunities that such technologies may afford ISR operations in the future, particularly in a coalition context where dynamic allocation of things to mission tasks can yield enormous advantages in mission agility and information availability and performance of human team members within these hybrid teams. Consequently, the focus has been on HCC and human-machine teaming with an emphasis on human-machine teaming and technologies with low training overheads.

The ultimate goal of exploiting IoT approaches in ISR will be to aid situational understanding defined as the 'product of applying analysis and judgment to the unit's situation awareness to determine the relationships of the factors present and form logical conclusions concerning threats to the force or mission accomplishment, opportunities for mission accomplishment, and gaps in information'. ${ }^{26}$ Situational understanding is intrinsically linked to information fusion in that it involves the collection and processing of data from multiple (coalition) sources as input, spanning multiple modalities. Achieving understanding highlights the importance of knowledge representation and reasoning - and the human user - in the information fusion process; i.e., it is an HCC task. Moreover, users' requirements for situational understanding place constraints on all levels of the fusion process, e.g., prioritizing and valuing of particular kinds of data, objects, contexts and intents. ${ }^{27}$ Dynamically and flexibly supporting these user requirements in an information fusion system for situational understanding requires a means for the user to interact with elements of the system at both relatively high and low levels.

The challenges involved in introducing IoT elements in such a system are enormous, suggesting that a new kind of 'cognitive thing' will be required, but the potential gains for future coalition operations are enormous.

\section{Acknowledgements}

This research was sponsored by the U.S. Army Research Laboratory and the UK Ministry of Defence under Agreement Number W911NF-16-3-0001. The views and conclusions contained in this document are those of the authors and should not be interpreted as representing the official policies, either expressed or implied, of the U.S. Army Research Laboratory, the U.S. Government, the UK Ministry of Defence or the UK Government. The U.S. and UK Governments are authorized to reproduce and distribute reprints for Government purposes notwithstanding any copy-right notation hereon.

\section{REFERENCES}

1. J. Gubbi, R. Buyya, S. Marusic, and M. Palaniswami, "Internet of things (iot): A vision, architectural elements, and future directions," Future Generation Computer Systems 29(7), pp. 1645-1660, 2013.

2. P. Fraga-Lamas, T. M. Fernández-Caramés, M. Suárez-Albela, L. Castedo, and M. González-López, "A review on internet of things for defense and public safety," Sensors 16(1644), 2016.

3. N. Suri, M. Tortonesi, J. Michaelis, P. Budulas, G. Benincasa, S. Russell, C. Stefanelli, and R. Winkler, "Analyzing the applicability of internet of things to the battlefield environment," in 2016 International Conference on Military Communications and Information Systems (ICMCIS), pp. 1-8, IEEE, 2016. 
4. M. V. Rasmussen, The Military's Business: Designing Military Power for the Future, Cambridge University Press, 2015.

5. E. Lawson and R. Barrons, "Warfare in the information age," The RUSI Journal 161(5), pp. 20-26, 2016.

6. T. Pham, G. Cirincione, D. Verma, and G. Pearson, "Intelligence, surveillance, and reconnaisance fusion for coalition operations," in Proc 11th International Conference on Information Fusion, 2008.

7. D. S. Alberts and R. E. Hayes, Power to the Edge: Command and Control in the Information Age, CCRP, 2003.

8. D. Norman, The Psychology of Everyday Things, Basic Books, 1988.

9. R. Crouser and R. Chang, "An affordance-based framework for human computation and human-computer collaboration," IEEE Transactions on Visualization and Computer Graphics 18(12), pp. 2859-2868, 2012.

10. M. Cummings, "Man versus machine or man + machine?," IEEE Intelligent Systems 29, pp. 62-69, Sep/Oct 2014.

11. D. Rose, Enchanted Objects: Design, Human Desire, and the Internet of Things, Simon and Schuster, 2014.

12. P. Pirolli and S. Card, "The sensemaking process and leverage points for analyst technology as identified through cognitive task analysis," in Proceedings of International Conference on Intelligence Analysis, 2005.

13. A. Clark and D. Chalmers, "The extended mind," Analysis 58(1), pp. 7-19, 1998.

14. D. Laney, "3D data management: Controlling data volume, velocity, and variety," tech. rep., META Group, 2001.

15. D. Verma, G. Bent, and I. Taylor, "Towards a distributed federated brain architecture using cognitive IoT devices," in 9th International Conference on Advanced Cognitive Technologies and Applications (COGNITIVE'17), 2017.

16. A. Preece, D. Braines, D. Pizzocaro, and C. Parizas, "Human-machine conversations to support multiagency missions," ACM SIGMOBILE Mobile Computing and Communications Review 18(1), pp. 75-84, 2014.

17. M. K. Dhami, D. R. Mandel, B. A. Mellers, and P. E. Tetlock, "Improving intelligence analysis with decision science," Perspectives on Psychological Science 10(6), pp. 753-757, 2015.

18. A. Preece, T. Norman, G. de Mel, D. Pizzocaro, M. Sensoy, and T. Pham, "Agilely assigning sensing assets to mission tasks in a coalition context," IEEE Intelligent Systems Jan/Feb, pp. 57-63, 2013.

19. B. P. Gerkey and M. J. Mataric, "A formal analysis and taxonomy of task allocation in Multi-Robot systems," The International Journal of Robotics Research 23(9), p. 939, 2004.

20. D. Pizzocaro, A. Preece, F. Chen, T. L. Porta, and A. Bar-Noy, "A distributed architecture for heterogeneous multi sensor-task allocation," in Proc 7th IEEE International Conference on Distributed Computing in Sensor Systems (DCOSS'11), 2011.

21. N. O'Leary, D. Braines, A. Preece, and W. Webberley, "Conversational homes," in 9th International Conference on Advanced Cognitive Technologies and Applications (COGNITIVE'17), 2017.

22. A. Preece, W. Webberley, D. Braines, E. G. Zaroukian, and J. Z. Bakdash, "Human computer collaboration at the edge: Enhancing collective situation understanding with controlled natural language," in ICCRTS 2016: 21st International Command and Control Research and Technology Symposium, 2016.

23. A. Preece, C. Gwilliams, C. Parizas, D. Pizzocaro, J. Z. Bakdash, and D. Braines, "Conversational sensing," in Proc Next-Generation Analyst II (SPIE Vol 9122), SPIE, 2014.

24. A. Toniolo, D. Braines, A. Preece, W. Webberley, T. J. Norman, P. Sullivan, and T. Dropps, "Conversational intelligence analysis," in 17th International Conference on Distributed Computing and Networking, 2016.

25. C. Parizas, D. Pizzocaro, A. Preece, and P. Zerfos, "Managing ISR sharing policies at the network edge using Controlled English," in Proc Ground/Air Multisensor Interoperability, Integration, and Networking for Persistent ISR IV (SPIE Vol 8742), SPIE, 2013.

26. B. C. Dostal, "Enhancing situational understanding through the employment of unmanned aerial vehicles," Army Transformation Taking Shape... Interim Brigade Combat Team Newsletter 01-18, 2007.

27. E. Blasch, "Level 5 (user refinement) issues supporting information fusion management," in 9th International Conference on Information Fusion, 2006. 